\title{
OPEN Serum FGF21 levels are altered by various factors including lifestyle behaviors in male subjects
}

\author{
Kaori Nakanishi ${ }^{凶}$, Chisaki Ishibashi, Seiko Ide, Ryohei Yamamoto, Makoto Nishida, \\ Izumi Nagatomo, Toshiki Moriyama \& Keiko Yamauchi-Takihara
}

Fibroblast growth factor (FGF) 21 has various functions, including glucose and lipid metabolism. This cross-sectional study aimed to investigate specific conditions that might influence the functions of FGF21. 398 men who underwent a health examination were enrolled in this study. Physical and biochemical parameters and information on several lifestyle behaviors were obtained from all subjects. FGF21 levels correlated with age, body mass index (BMI), waist circumference (WC), systolic blood pressure (SBP), diastolic blood pressure (DBP), aspartate aminotransferase (AST), alanine aminotransferase (ALT), gamma-glutamyl transpeptidase ( $\gamma$-GTP), uric acid, total cholesterol (TC), triglycerides (TG), high-density lipoprotein cholesterol (HDLC), fasting plasma glucose (FPG), and HbA1c. Moreover, FGF21 levels were significantly associated with lifestyle behaviors, including smoking status and breakfast and alcohol consumption frequency. Multivariable regression analysis showed that age, ALT, Y-GTP, smoking status, and breakfast and alcohol consumption frequency were independent variables for FGF21 levels. Assessment among the non-obese and obese groups showed that FGF21 levels correlated with WC, SBP, and TC only in the non-obese group. Thus, serum FGF21 levels were affected by several factors, including lifestyle behaviors, age, and liver function. To assess the functions of FGF21 in individuals, considering these factors would be essential.

Fibroblast growth factor (FGF) 21 is predominantly derived from the liver and is a member of the FGF19 subfamily, together with FGF15/19 and FGF23. Unlike other FGF subfamilies, members of the FGF19 subfamily exhibit hormone-like functions and require Klotho proteins: $\alpha$ Klotho and $\beta \mathrm{Klotho}$, as co-factors to increase their binding affinity to FGF receptors ${ }^{1}$. FGF21 interacts with $\beta$ Klotho and plays an important role in glucose and lipid metabolism. It regulates glucose uptake in adipocytes, enhances fatty acid oxidation in the liver, and inhibits lipogenesis ${ }^{2,3}$.

As FGF21 administration in obese animal models has shown an improvement in insulin sensitivity, a decrease in triglyceride and cholesterol levels, and a reduction in adiposity, FGF21 is expected as a new therapy for obesity and obesity-related diseases, including type 2 diabetes and nonalcoholic steatohepatitis ${ }^{4-6}$. Paradoxically, serum levels of FGF21 are increased in individuals with obesity, type 2 diabetes, and metabolic syndrome ${ }^{7,8}$. While this increase in FGF21 levels is regarded as a compensatory response or the FGF21-resistant state, the precise underlying mechanism for this increase remains unclear, ${ }^{9,10}$.

We previously reported that FGF21 levels were high in smokers and negatively correlated with the metabolic syndrome-related cytokine, adiponectin. Interestingly, FGF21 levels were differentially associated with liver function and total cholesterol among smokers and never-smokers, suggesting that smoking stress affects the relationship between FGF21 and metabolic parameters ${ }^{11}$. Moreover, we demonstrated a sex-based difference in the relationship between FGF21 levels and metabolic parameters ${ }^{12}$. These results suggest that there may be some conditions that affect FGF21 levels.

Since FGF21 has various functions in multiple target organs, FGF21 biology is complicated and several uncertainties remain ${ }^{13}$. Therefore, in the present study, we focused on evaluating specific conditions that might influence the functions of FGF21 by assessing the associations of serum levels of FGF21 with physical parameters, biochemical parameters, and lifestyle behaviors using cross-sectional data of male subjects. 


\begin{tabular}{|l|l|}
\hline $\mathrm{n}$ & 398 \\
\hline Age $(\mathrm{years})$ & $42(37-49)$ \\
\hline BMI $\left(\mathrm{kg} / \mathrm{m}^{2}\right)$ & $24.3(21.9-9.3)$ \\
\hline $\mathrm{WC}(\mathrm{cm})$ & $86.0(77.5-96.0)$ \\
\hline SBP $(\mathrm{mmHg})$ & $124(112-133)$ \\
\hline DBP $(\mathrm{mmHg})$ & $78(71-87)$ \\
\hline AST $(\mathrm{IU} / \mathrm{l})$ & $23(18-32)$ \\
\hline ALT $(\mathrm{IU} / \mathrm{l})$ & $28(17-47)$ \\
\hline$\gamma$-GTP $(\mathrm{IU} / \mathrm{l})$ & $39(23-69)$ \\
\hline Cr $(\mathrm{mg} / \mathrm{dl})$ & $0.9 \pm 0.1$ \\
\hline UA $(\mathrm{mg} / \mathrm{dl})$ & $6.3 \pm 1.2$ \\
\hline TC $(\mathrm{mg} / \mathrm{dl})$ & $201 \pm 32$ \\
\hline TG $(\mathrm{mg} / \mathrm{dl})$ & $105(70-158)$ \\
\hline HDLC $(\mathrm{mg} / \mathrm{dl})$ & $52(44-61)$ \\
\hline FPG $(\mathrm{mg} / \mathrm{dl})$ & $88(83-94)$ \\
\hline HbAlc $(\%)$ & $5.3(5.1-5.5)$ \\
\hline FGF21 $(\mathrm{pg} / \mathrm{ml})$ & $165(106-256)$ \\
\hline
\end{tabular}

Table 1. Characteristics of the study subjects. Data are expressed as means \pm SD or medians (interquartile range). BMI, body mass index; WC, waist circumference; SBP, systolic blood pressure; DBP, diastolic blood pressure; AST, aspartate aminotransferase; ALT, alanine aminotransferase; $\gamma$-GTP, gamma-glutamyl transpeptidase; Cr, creatinine; UA, uric acid; TC, total cholesterol; TG, triglycerides; HDLC, high-density lipoprotein cholesterol; FPG, fasting plasma glucose; FGF21, fibroblast growth factor 21.

\begin{tabular}{|l|l|l|}
\hline & \multicolumn{1}{|l|}{} & $\mathbf{P}$ \\
\hline Age & $0.24^{*}$ & $<0.0001$ \\
\hline BMI & $0.13^{*}$ & 0.0001 \\
\hline WC & $0.15^{*}$ & $<0.0001$ \\
\hline SBP & $0.20^{*}$ & $<0.0001$ \\
\hline DBP & $0.21^{*}$ & $<0.0001$ \\
\hline AST & $0.19^{*}$ & $<0.0001$ \\
\hline ALT & $0.20^{*}$ & $<0.0001$ \\
\hline$\gamma$-GTP & $0.31^{*}$ & $<0.0001$ \\
\hline Cr & -0.02 & 0.571 \\
\hline UA & $0.17^{*}$ & $<0.0001$ \\
\hline TC & $0.10^{*}$ & 0.004 \\
\hline TG & $0.23^{*}$ & $<0.0001$ \\
\hline HDLC & $-0.10^{*}$ & 0.003 \\
\hline FPG & $0.17^{*}$ & $<0.0001$ \\
\hline HbA1c & $0.13^{*}$ & 0.0001 \\
\hline
\end{tabular}

Table 2. Correlations of fibroblast growth factor 21 levels with physical and biochemical parameters. ${ }^{\star} \mathrm{P}<0.005$. Abbreviations are as in Table 1.

\section{Results}

Association of FGF21 levels with each parameter. The characteristics of the study participants are presented in Table 1 . The median age and FGF21 levels were $42(37-49)$ years and $165(106-256) \mathrm{pg} / \mathrm{mL}$, respectively.

Table 2 shows the correlations between serum levels of FGF21 and each physical and biochemical parameter. Serum levels of FGF21 showed significant positive correlations with age, Body mass index (BMI: body weight $[\mathrm{kg}]$ divided by squared height $\left[\mathrm{m}^{2}\right]$ ), waist circumference (WC), systolic blood pressure (SBP), diastolic blood pressure (DBP), aspartate aminotransferase (AST), alanine aminotransferase (ALT), gamma-glutamyl transpeptidase ( $\gamma$-GTP), uric acid (UA), total cholesterol (TC), triglycerides (TG), fasting plasma glucose (FPG), and $\mathrm{HbA1c}$ and a negative correlation with high-density lipoprotein cholesterol (HDLC).

Smoking status, breakfast and alcohol consumption affect serum levels of FGF21. As we previously reported that serum levels of FGF21 were upregulated in smokers ${ }^{11,12}$, FGF21 levels were significantly higher in smokers [210 (124-353) pg/mL] than in never smokers [147 (101-224) pg/mL, p<0.0001] and posi- 
tively correlated with smoking status $(\tau=0.14, \mathrm{p}<0.0001)$. Moreover, serum levels of FGF21 significantly negatively correlated with breakfast consumption frequency $(\tau=-0.12, p<0.0001)$, and positively correlated with alcohol consumption frequency $(\tau=0.15, \mathrm{p}<0.0001)$ and daily alcohol intake $(\tau=0.08, \mathrm{p}=0.0237)$. We further assessed the associations of FGF21 levels with breakfast and alcohol consumption, as shown in Fig. 1. Serum levels of FGF21 were significantly increased in subjects whose breakfast consumption frequency was low (Fig. 1A). In addition, FGF21 levels were significantly increased in subjects whose alcohol consumption frequency was high (Fig. 1B) and tended to increase in subjects whose daily alcohol intake was high (Fig. 1C). These results suggest that lifestyle behaviors, including breakfast consumption and alcohol consumption affected the serum levels of FGF21 as smoking status.

Age strongly associates with serum levels of FGF21. Multivariable regression analysis showed that age, ALT, $\gamma$-GTP, smoking status, and breakfast and alcohol consumption frequency were independent variables for FGF21 levels (Table 3). As correlation and regression analyses showed that age is a strong variable for FGF21 levels, we assessed FGF21 levels in each age group. The median serum levels of FGF21 in participants of age $30-39$ years $(n=129), 40-49$ years $(n=175)$, and $\geq 50$ years $(n=94)$ were $119(81-201) p g / m L, 181(117-271)$ $\mathrm{pg} / \mathrm{mL}, 214$ (150-359) pg/mL, respectively. Significant differences were observed among age groups $(\mathrm{p}=0.0001)$.

Relationship between FGF21 and each parameter in non-obese and obese group. FGF21 is considered a potent inhibitor of obesity and FGF21 therapy has been identified as a possible treatment for obesity and obesity-related diseases in recent years ${ }^{5}$. Since FGF21 show a strong association with obesity, we categorized the study participants into non-obese $\left(\mathrm{BMI}<25 \mathrm{~kg} / \mathrm{m}^{2}\right)$ and obese $\left(\mathrm{BMI} \geq 25 \mathrm{~kg} / \mathrm{m}^{2}\right)$ groups and assessed the association between FGF21 levels and each parameter in both groups (Supplementary Table S1 online and Table 4).

Serum levels of FGF21 showed significant positive correlations with WC, SBP, and TC only in the non-obese group, while the positive correlation between FGF21 and HbA1c was observed only in the obese group. Thus, it can be suggested that FGF21 effects may differ depending on the BMI of each subject.

\section{Discussion}

FGF21 has multiple functions, and recent studies have shown its various protective effects. Our previous study showed that FGF21 levels correlated with adiponectin, a metabolic syndrome-related cytokine that also has protective effects, including anti-inflammatory, anti-atherogenic, and anti-diabetic effects ${ }^{11,14}$. Since FGF21 regulates oxidative stress and attenuates inflammation, it is considered a stress-response factor ${ }^{15,16}$. In the present study, we analyzed the association of FGF21 levels with metabolic parameters and several lifestyle behaviors. We previously reported that smoking status affects the serum levels of FGF2 $1^{11}$. In this study, we demonstrated that not only smoking, but also breakfast and alcohol consumption frequency affected the FGF21 levels. FGF21 has been reported as one of the genetic variants for breakfast skipping ${ }^{17}$, suggesting the strong association between breakfast consumption frequency and FGF21. Breakfast is considered the most important meal of the day and affects physical and mental health ${ }^{18,19}$. It is reported that breakfast skipping is associated with inflammation, related to an increase in WC and low-density lipoprotein cholesterol, and cardiovascular disease risk ${ }^{20-23}$. We found FGF21 levels to be increased in subjects with a low breakfast consumption frequency. Moreover, increased FGF21 levels were shown in subject with a high alcohol consumption frequency. FGF21 shows a protective effect on the liver damage induced by alcohol. In animal models, FGF21 administration reduces alcohol intake via the central nervous system interacting with $\beta K l_{\text {otho }}{ }^{15,24}$. An increase in FGF21 levels in a stressed condition, including smoking, breakfast skipping, and alcohol ingestion, is suggested to be a protective response of FGF21.

Serum levels of FGF21 is known to have a large variation even in healthy population ${ }^{25}$. As presented in this study, the FGF21 levels might have been affected by some unknown factors, including lifestyle behaviors of each individual. Since FGF21 is considered a stress-response factor, it is quite possible that increased FGF21 levels are implicating the stressed condition. To our knowledge, there are no prior studies that address the influence of lifestyle behaviors on FGF21 levels, further studies are definitively needed to clarify these relationships.

While serum levels of FGF21 were related to many parameters, we found that age was a strong factor affecting FGF21 levels. Evaluation of serum levels of FGF21 in each age group showed that FGF21 levels were significantly increased along with aging. Aging is associated with various diseases, including cardiovascular diseases, cancer, and pulmonary diseases, which are known to be the leading causes of death worldwide ${ }^{26}$. Moreover, obesity and obesity-related diseases are associated with aging ${ }^{27}$. Previous studies have reported that FGF21 extended the lifespan of mice and showed a protective effect on age-related changes ${ }^{28,29}$. Since FGF21 can ameliorate both aging and metabolism, FGF21 is regarded as a key factor linking aging and metabolism ${ }^{30}$. In the present study, although the subjects were individuals who did not present any signs of disease, FGF21 levels were upregulated in the aged groups. Considering the anti-aging effects of FGF21, these increased FGF21 levels might be a compensatory response toward progress in aging and age-related changes. Since the age range was limited to median age $42(37-49)$ years in the present study, we are willing to assess aged population in our further studies to elucidate this anti-aging effects of FGF21.

Individuals with obesity, in whom protective effects are not shown with increased serum levels of FGF21, are considered FGF21-resistant ${ }^{7,9}$. To evaluate differential functions of FGF21 between non-obese and obese subjects, we assessed the association of FGF21 with metabolic parameters among both groups. As previously reported, FGF21 levels were significantly high in the obese group. Serum levels of FGF21 correlated with more variables in the non-obese group than in the obese group. Moreover, WC, SBP, and TC correlated with FGF21 only in the non-obese group. WC is a known parameter for the definition of metabolic syndrome and is strongly associated with visceral fat accumulation ${ }^{31}$. Although both WC and FGF21 levels were high in obese subjects, there was 


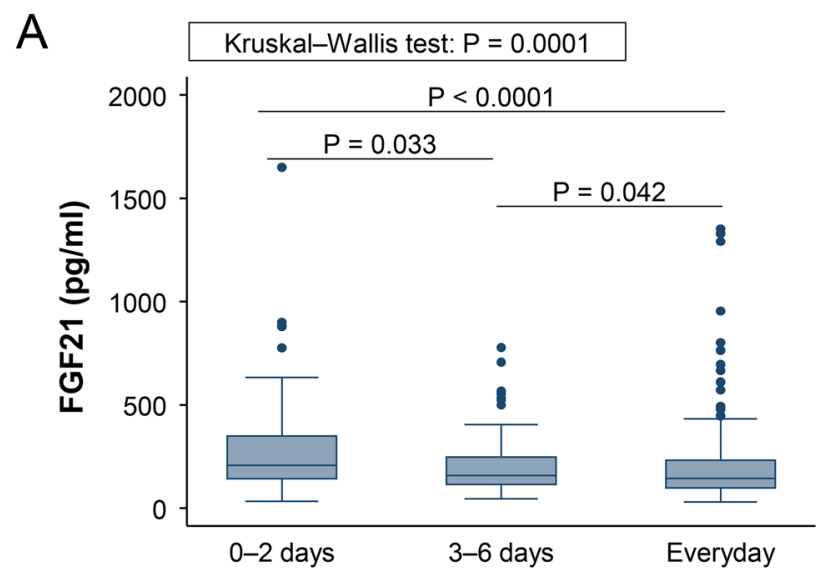

Breakfast consumption (per week)

B

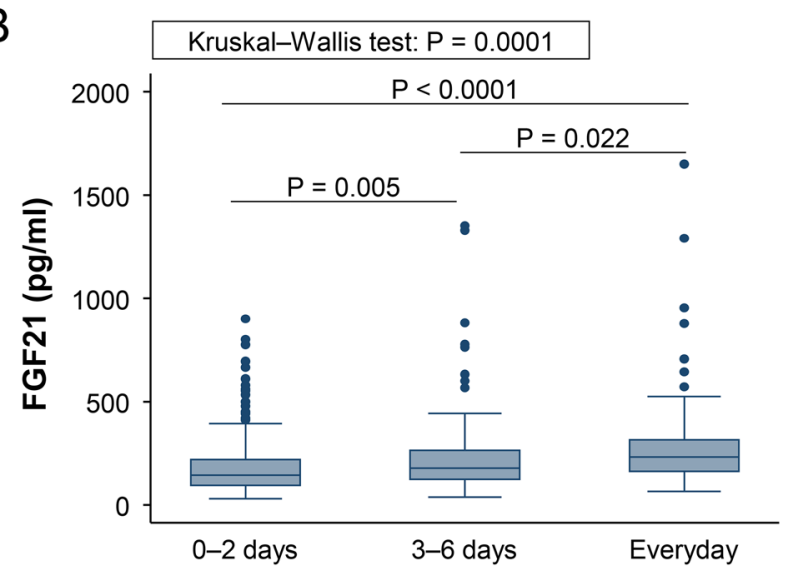

Alcohol consumption (per week)

C

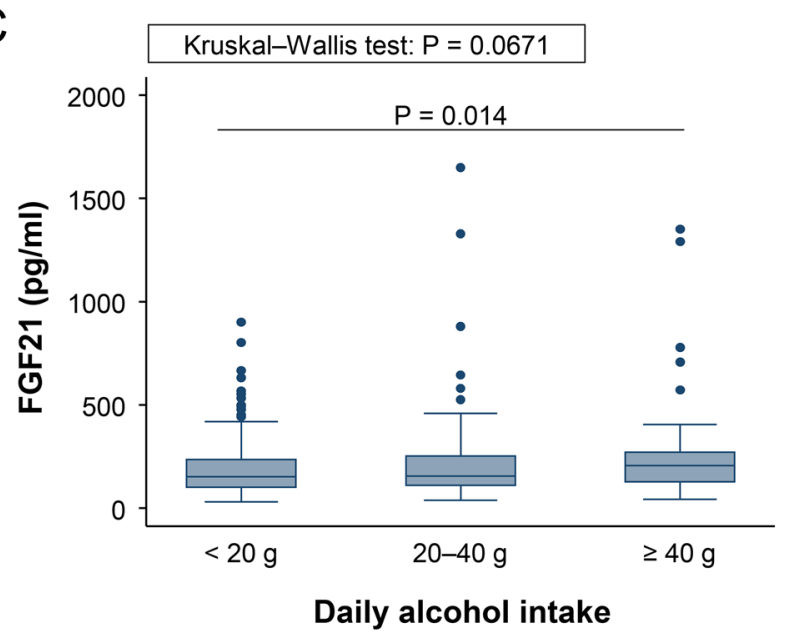

Figure 1. Serum levels of fibroblast growth factor (FGF) 21 are affected by breakfast and alcohol consumption. Associations of serum levels of FGF21 with (A) breakfast consumption frequency ( $0-2$ days a week: $n=79$, 3-6 days a week: $n=69$, everyday: $n=240),(B)$ alcohol consumption frequency $(0-2$ days a week: $n=250$, 3-6 days a week: $n=73$, everyday: $n=75)$, and $(C)$ daily alcohol intake $(<20 \mathrm{~g}: \mathrm{n}=170,20-40 \mathrm{~g}: \mathrm{n}=78, \geq 40 \mathrm{~g}$ : $\mathrm{n}=42$ ). Kruskal-Wallis test with Dunn's post-hoc test was used to test between group differences. Data are shown as the sample minimum, lower quartile, median, upper quartile, and sample maximum. Dots represent outliers. 


\begin{tabular}{|l|l|l|}
\hline & \multicolumn{1}{|l|}{$\boldsymbol{\beta}$} & $\mathbf{P}$ \\
\hline Age & $0.0108^{*}$ & $<0.0001$ \\
\hline WC & 0.0008 & 0.634 \\
\hline DBP & 0.0011 & 0.456 \\
\hline ALT & $0.0011^{*}$ & 0.042 \\
\hline$\gamma$-GTP & $0.0006^{*}$ & 0.049 \\
\hline UA & 0.0144 & 0.256 \\
\hline TC & 0.0001 & 0.829 \\
\hline TG & 0.0002 & 0.234 \\
\hline HDLC & -0.0013 & 0.339 \\
\hline FPG & 0.0012 & 0.378 \\
\hline HbA1c & -0.0326 & 0.415 \\
\hline Smoking status & $0.0678^{*}$ & 0.022 \\
\hline Breakfast consumption frequency & $-0.0585^{*}$ & 0.001 \\
\hline Alcohol consumption frequency & $0.0795^{*}$ & $<0.0001$ \\
\hline
\end{tabular}

Table 3. Multivariable regression analysis of log-transformed fibroblast growth factor $21 .{ }^{\star} \mathrm{P}<0.05$. Abbreviations are as in Table 1.

\begin{tabular}{|c|c|c|c|c|}
\hline & \multicolumn{2}{|c|}{ Non-obese } & \multicolumn{2}{|l|}{ Obese } \\
\hline & \multicolumn{2}{|c|}{$\mathrm{BMI}<25 \mathrm{~kg} / \mathrm{m}^{2}$} & \multicolumn{2}{|c|}{$\mathrm{BMI} \geq 25 \mathrm{~kg} / \mathrm{m}^{2}$} \\
\hline & $\tau$ & $\mathbf{P}$ & $\tau$ & $\mathbf{P}$ \\
\hline Age & $0.27^{\star}$ & $<0.0001$ & $0.17^{\star}$ & 0.001 \\
\hline BMI & 0.09 & 0.058 & -0.04 & 0.486 \\
\hline WC & $0.14^{*}$ & 0.002 & 0.00 & 0.965 \\
\hline SBP & $0.21^{\star}$ & $<0.0001$ & 0.05 & 0.298 \\
\hline DBP & $0.21^{\star}$ & $<0.0001$ & $0.12^{\star}$ & 0.015 \\
\hline AST & $0.16^{*}$ & 0.0004 & $0.13^{*}$ & 0.011 \\
\hline ALT & $0.16^{*}$ & 0.0004 & $0.12^{\star}$ & 0.022 \\
\hline$\gamma$-GTP & $0.31^{\star}$ & $<0.0001$ & $0.25^{\star}$ & $<0.0001$ \\
\hline $\mathrm{Cr}$ & -0.04 & 0.379 & 0.01 & 0.911 \\
\hline UA & $0.12^{*}$ & 0.011 & $0.12^{*}$ & 0.018 \\
\hline TC & $0.09^{*}$ & 0.040 & 0.07 & 0.182 \\
\hline TG & $0.14^{\star}$ & 0.002 & $0.24^{*}$ & $<0.0001$ \\
\hline HDLC & -0.02 & 0.593 & -0.04 & 0.388 \\
\hline FPG & $0.11^{\star}$ & 0.014 & $0.16^{*}$ & 0.001 \\
\hline $\mathrm{HbAlc}$ & 0.04 & 0.349 & $0.13^{*}$ & 0.012 \\
\hline
\end{tabular}

Table 4. Correlations of fibroblast growth factor 21 with physical and biochemical parameters in the nonobese and obese groups. ${ }^{\star} \mathrm{P}<0.05$. Abbreviations are as in Table 1 .

no correlation between WC and FGF21. This disrupted relationship between FGF21 and WC suggests that the protective effects of FGF21 are attenuated in obese subjects. We previously reported that serum levels of FGF21 correlated with metabolic parameters differently among smokers and never-smokers ${ }^{11}$. We also confirmed that there were sex differences in the relationship between FGF21 levels and metabolic parameters ${ }^{12}$. These results show a variable relationship between FGF21 levels and metabolic parameters and that the effect of FGF21 may vary depending on the individual background. Since FGF21 is expected as a new therapy for obesity and obesityrelated diseases ${ }^{4,5}$, effective conditions are needed to be evaluated.

FGF21 levels have been reported to be altered in various diseases ${ }^{7,8,32}$. However, as demonstrated in the present study, the serum levels of FGF21 are affected by several factors. Therefore, to assess the functions of FGF21 in subjects with such diseases, considering these factors and matching the conditions among the subjects is essential. Furthermore, as the mechanisms of change in FGF21 levels are still unclear, we believe that our findings might help elucidate the complicated biology of FGF21.

This study has some limitations. Since the study was exploratory research to find the FGF21 levels affecting factors, the sample size was not calculated in advance. We performed the post-hoc power analyses of our results: associations of FGF21 levels with breakfast consumption and alcohol consumption (Fig. 1) and multiple regression analysis (Table 3), and confirmed that the sample size in the study was adequate. Another limitation is that 
the study was a single center study with limited age range of subjects. Multicenter study with broad age range of subjects is preferable in the future study.

In conclusion, we evaluated the parameters and lifestyle behaviors that may influence FGF21 functions in the present study. Serum levels of FGF21 were affected by several lifestyle behaviors, including smoking status, breakfast consumption, and alcohol consumption. Moreover, in the FGF21 relating parameters, age was strongly associated with FGF21 levels. Serum levels of FGF21 are associated with metabolic parameters differently in non-obese and obese subjects.

\section{Methods}

Study subjects. This study included cross-sectional data obtained from employees at Osaka University. The subjects were randomly selected individuals who underwent an annual health examination at the Osaka University Health and Counseling Center. The inclusion and exclusion criteria for this study were as follows: (1) male subjects, (2) with no underlying diseases (3) had not taken any chronic or frequent medication for at least 1 year before their health examination, and (4) had not experienced any acute illness within the previous 2 weeks. A total of 398 Japanese men were enrolled in the study. This study was carried out in accordance with the Declaration of Helsinki and the Ethics Guidelines for Clinical Research from the Ministry of Health, Labour and Welfare and the Ministry of Education, Culture, Sports, Science and Technology. All experimental protocols in this study were approved by the Ethics Committee of Health and Counseling Center, Osaka University, and written informed consent was obtained from all subjects prior to participation in the study.

Physical and biochemical parameters. BMI, WC at the umbilical level, SBP, DBP were measured as physical parameters.

Serum was collected from subjects between 9 and 11 A.M. after an overnight fast and stored at $\leq-20^{\circ} \mathrm{C}$ until assayed. Serum concentrations of AST, ALT, $\gamma$-GTP, creatinine, UA, TC, TG, HDLC, FPG, HbA1c, and FGF21 were measured as biochemical parameters from same blood sample. Serum levels of FGF21 were measured in duplicate for each sample using a commercially available sandwich enzyme-linked immunoassay system (DF2100; R\&D Systems Inc., Minneapolis, USA) with assay range: 31.3-2000 pg/ml, intra-assay CV: 2.9-3.9\%, and interassay CV: $5.2-10.9 \%$. The methodology was performed according to the manufacturer's instructions ${ }^{11,15,33}$.

Lifestyle assessments. Information on the medical history, current treatments, smoking status, and lifestyle behaviors of the study participants were obtained via questionnaires. Each information was reconfirmed through expert interviews by trained nurses. Smoking status was semi-quantified as $0=$ never smoker and $1=$ smoker. Lifestyle behaviors, including breakfast and alcohol consumption frequency, and daily alcohol intake, were asked as follows, and each answer was semi-quantified using the following scales. Breakfast consumption frequency: "How many days a week do you eat breakfast?" on three scales: $1=0-2$ days a week, $2=3-6$ days a week, 3 =everyday; alcohol consumption frequency: "How many days a week do you drink alcohol?" on three scales: 1 =0-2 days a week, 2=3-6 days a week, 3 =everyday; daily alcohol intake: "How many amounts of pure alcohol do you have on a typical day when you are drinking?" on three scales: $1=<20 \mathrm{~g}, 2=20-40 \mathrm{~g}, 3=\geq 40 \mathrm{~g}$.

Statistical analyses. All statistical analyses were performed using STATA 14 (STATA Corp LLC, College Station, TX, USA). The distribution of continuous variables was tested using the Shapiro-Wilk test. Normally distributed variables are presented as means \pm standard deviation; non-normally distributed variables are reported as medians with interquartile ranges. Student's t-test or the Mann-Whitney U test was used to compare differences between the two groups. Kendall's rank correlation coefficient and multiple regression analysis were used to analyze the variables. For multi-group comparisons, the Kruskal-Wallis test with Dunn's post-hoc test was used. Statistical significance was set at $\mathrm{P}<0.05$.

\section{Data availability}

The datasets generated during and/or analyzed during the current study are available from the corresponding author on reasonable request.

Received: 10 September 2021; Accepted: 9 November 2021

Published online: 19 November 2021

\section{References}

1. Kuro-o, M. Endocrine FGFs and Klothos: Emerging concepts. Trends Endocrinol. Metab. 19, 239-245. https://doi.org/10.1016/j. tem.2008.06.002 (2008).

2. Kharitonenkov, A. et al. FGF-21 as a novel metabolic regulator. J. Clin. Investig. 115, 1627-1635. https://doi.org/10.1172/JCI23606 (2005).

3. Ding, X. et al. betaKlotho is required for fibroblast growth factor 21 effects on growth and metabolism. Cell Metab. 16, 387-393. https://doi.org/10.1016/j.cmet.2012.08.002 (2012).

4. Geng, L., Lam, K. S. L. \& Xu, A. The therapeutic potential of FGF21 in metabolic diseases: From bench to clinic. Nat. Rev. Endocrinol. 16, 654-667. https://doi.org/10.1038/s41574-020-0386-0 (2020).

5. Jimenez, V. et al. FGF21 gene therapy as treatment for obesity and insulin resistance. EMBO Mol. Med. 10, e8791. https://doi.org/ 10.15252/emmm.201708791 (2018).

6. Morris, A. Potential treatment strategy for NASH. Nat. Rev. Endocrinol. 15, 129. https://doi.org/10.1038/s41574-019-0162-1 (2019).

7. Zhang, X. et al. Serum FGF21 levels are increased in obesity and are independently associated with the metabolic syndrome in humans. Diabetes 57, 1246-1253. https://doi.org/10.2337/db07-1476 (2008).

8. Cheng, X., Zhu, B., Jiang, F. \& Fan, H. Serum FGF-21 levels in type 2 diabetic patients. Endocr. Res. 36, 142-148. https://doi.org/ $10.3109 / 07435800.2011 .558550(2011)$. 
9. Fisher, F. M. et al. Obesity is a fibroblast growth factor 21 (FGF21)-resistant state. Diabetes 59, 2781-2789. https://doi.org/10.2337/ db10-0193 (2010).

10. Li, H. et al. Fibroblast growth factor 21 increases insulin sensitivity through specific expansion of subcutaneous fat. Nat. Commun. 9, 272. https://doi.org/10.1038/s41467-017-02677-9 (2018).

11. Nakanishi, K. et al. Klotho-related molecules upregulated by smoking habit in apparently healthy men: A cross-sectional study. Sci. Rep. 5, 14230. https://doi.org/10.1038/srep14230 (2015).

12. Nakanishi, K. et al. An implication of Klotho-related molecules in different smoking-related health outcomes between men and women. Clin. Chim. Acta 476, 44-48. https://doi.org/10.1016/j.cca.2017.11.007 (2018).

13. Kharitonenkov, A. \& DiMarchi, R. Fibroblast growth factor 21 night watch: Advances and uncertainties in the field. J. Intern. Med. 281, 233-246. https://doi.org/10.1111/joim.12580 (2017).

14. Okamoto, Y., Kihara, S., Funahashi, T., Matsuzawa, Y. \& Libby, P. Adiponectin: A key adipocytokine in metabolic syndrome. Clin. Sci. 110, 267-278. https://doi.org/10.1042/CS20050182 (2006).

15. Desai, B. N. et al. Fibroblast growth factor 21 (FGF21) is robustly induced by ethanol and has a protective role in ethanol associated liver injury. Mol. Metab. 6, 1395-1406. https://doi.org/10.1016/j.molmet.2017.08.004 (2017).

16. Gomez-Samano, M. A. et al. Fibroblast growth factor 21 and its novel association with oxidative stress. Redox Biol. 11, 335-341. https://doi.org/10.1016/j.redox.2016.12.024 (2017).

17. Dashti, H. S. et al. Genome-wide association study of breakfast skipping links clock regulation with food timing. Am. J. Clin. Nutr. 110, 473-484. https://doi.org/10.1093/ajcn/nqz076 (2019).

18. Timlin, M. T. \& Pereira, M. A. Breakfast frequency and quality in the etiology of adult obesity and chronic diseases. Nutr. Rev. 65, 268-281. https://doi.org/10.1301/nr.2007.jun.268-281 (2007).

19. Pengpid, S. \& Peltzer, K. Skipping breakfast and its association with health risk behaviour and mental health among university students in 28 countries. Diabetes Metab. Syndr. Obes. 13, 2889-2897. https://doi.org/10.2147/DMSO.S241670 (2020).

20. Bonnet, J. P., Cardel, M. I., Cellini, J., Hu, F. B. \& Guasch-Ferre, M. Breakfast skipping, body composition, and cardiometabolic risk: A systematic review and meta-analysis of randomized trials. Obesity 28, 1098-1109. https://doi.org/10.1002/oby.22791 (2020).

21. Smith, K. J. et al. Skipping breakfast: Longitudinal associations with cardiometabolic risk factors in the Childhood Determinants of Adult Health Study. Am. J. Clin. Nutr. 92, 1316-1325. https://doi.org/10.3945/ajen.2010.30101 (2010).

22. Cahill, L. E. et al. Prospective study of breakfast eating and incident coronary heart disease in a cohort of male US health professionals. Circulation 128, 337-343. https://doi.org/10.1161/CIRCULATIONAHA.113.001474 (2013).

23. Zhu, S. et al. Habitually skipping breakfast is associated with chronic inflammation: A cross-sectional study. Public Health Nutr. 24, 2936-2943. https://doi.org/10.1017/S1368980020001214 (2021).

24. Soberg, S. et al. FGF21, a liver hormone that inhibits alcohol intake in mice, increases in human circulation after acute alcohol ingestion and sustained binge drinking at Oktoberfest. Mol. Metab. 11, 96-103. https://doi.org/10.1016/j.molmet.2018.03.010 (2018).

25. Galman, C. et al. The circulating metabolic regulator FGF21 is induced by prolonged fasting and PPARalpha activation in man. Cell Metab. 8, 169-174. https://doi.org/10.1016/j.cmet.2008.06.014 (2008).

26. Jaul, E. \& Barron, J. Age-related diseases and clinical and public health implications for the 85 years old and over population. Front. Public Health 5, 335. https://doi.org/10.3389/fpubh.2017.00335 (2017).

27. Frasca, D., Blomberg, B. B. \& Paganelli, R. Aging, obesity, and inflammatory age-related diseases. Front. Immunol. 8, 1745. https:// doi.org/10.3389/fimmu.2017.01745 (2017).

28. Youm, Y. H., Horvath, T. L., Mangelsdorf, D. J., Kliewer, S. A. \& Dixit, V. D. Prolongevity hormone FGF21 protects against immune senescence by delaying age-related thymic involution. Proc. Natl. Acad. Sci. USA 113, 1026-1031. https://doi.org/10.1073/pnas. $1514511113(2016)$.

29. Inagaki, T. et al. Inhibition of growth hormone signaling by the fasting-induced hormone FGF21. Cell Metab. 8, 77-83. https:// doi.org/10.1016/j.cmet.2008.05.006 (2008).

30. Yan, J. et al. The roles and pharmacological effects of FGF21 in preventing aging-associated metabolic diseases. Front Cardiovasc. Med. 8, 655575. https://doi.org/10.3389/fcvm.2021.655575 (2021).

31. Katzmarzyk, P. T., Janssen, I., Ross, R., Church, T. S. \& Blair, S. N. The importance of waist circumference in the definition of metabolic syndrome: Prospective analyses of mortality in men. Diabetes Care 29, 404-409. https://doi.org/10.2337/diacare.29.02. 06.dc05-1636 (2006).

32. Wu, L. et al. Fibroblast growth factor 21 is related to atherosclerosis independent of nonalcoholic fatty liver disease and predicts atherosclerotic cardiovascular events. J. Am. Heart Assoc. 9, e015226. https://doi.org/10.1161/JAHA.119.015226 (2020).

33. Hanssen, M. J. et al. Serum FGF21 levels are associated with brown adipose tissue activity in humans. Sci. Rep. 5, 10275. https:// doi.org/10.1038/srep10275 (2015).

\section{Acknowledgements}

The authors thank all the nurses and technicians who helped collect the data. Similarly, the authors would like to express our appreciation to all study participants.

\section{Author contributions}

K.N., I.N., and K.Y-T. designed the study. K.N. performed the experiments, analyzed the data, and wrote the manuscript. C.I., I.N., and K.Y-T. provided appropriate suggestions during the development of this study. Valuable comments on a first draft were received from S.I., R.Y., and T.M. All authors have given approval to the final version of the manuscript.

\section{Funding}

This work was supported by JSPS KAKENHI (Grant Number JP18K17923).

\section{Competing interests}

The authors declare no competing interests.

\section{Additional information}

Supplementary Information The online version contains supplementary material available at https://doi.org/ 10.1038/s41598-021-02075-8.

Correspondence and requests for materials should be addressed to K.N.

Reprints and permissions information is available at www.nature.com/reprints. 
Publisher's note Springer Nature remains neutral with regard to jurisdictional claims in published maps and institutional affiliations.

(c) (i) Open Access This article is licensed under a Creative Commons Attribution 4.0 International License, which permits use, sharing, adaptation, distribution and reproduction in any medium or format, as long as you give appropriate credit to the original author(s) and the source, provide a link to the Creative Commons licence, and indicate if changes were made. The images or other third party material in this article are included in the article's Creative Commons licence, unless indicated otherwise in a credit line to the material. If material is not included in the article's Creative Commons licence and your intended use is not permitted by statutory regulation or exceeds the permitted use, you will need to obtain permission directly from the copyright holder. To view a copy of this licence, visit http://creativecommons.org/licenses/by/4.0/.

(C) The Author(s) 2021 